\title{
Searching for neutrinos from dark matter annihilations in (dwarf) galaxies and galaxy clusters with IceCube
}

\section{The IceCube Collaboration ${ }^{\dagger}$,}

${ }^{\dagger}$ http://icecube.wisc.edu/collaboration/authors/icrc15_icecube

E-mail: meike.de.withedesv.de

In many models, the self-annihilation of dark matter particles will create neutrinos which can be detected on Earth. An excess flux of these neutrinos is expected from regions of increased dark matter density, like (dwarf) galaxies and galaxy clusters. The IceCube neutrino observatory, a cubic-kilometer neutrino detector at the South Pole, is capable of detecting neutrinos down to energies of the order of $10 \mathrm{GeV}$ and is therefore able to constrain the self-annihilation cross section as a function of the mass of the dark matter particle. This work will present the analysis method and results obtained from the first search for dark matter annihilations in (dwarf) galaxies and galaxy clusters with the complete IceCube detector.

Corresponding authors: M. de With ${ }^{* 1}$, E. Bernardini ${ }^{1}$

${ }^{1}$ DESY, D-15735 Zeuthen, Germany

The 34th International Cosmic Ray Conference,

30 July- 6 August, 2015

The Hague, The Netherlands

\footnotetext{
* Speaker.
} 


\section{Introduction}

There are many observations which imply the existence of a new type of non-baryonic, nonvisible and (meta)stable matter, usually called dark matter (for a review, see e.g. [四]). The currently most favoured candidates are Weakly Interacting Massive Particles (WIMPs), which are predicted by many theories for Beyond the Standard Model physics, for example supersymmetry and theories with extra dimensions. Typical masses are approximately between $10 \mathrm{GeV}$ and $100 \mathrm{TeV}$.

So far only the gravitational effects of dark matter have been detected, but many experiments are searching for other signatures, both to confirm the gravitational observations and to study the properties of this new type of matter, which are so far mainly unknown. For example, indirect detection experiments search for a flux of stable particles produced in the self-annihilations or decays of WIMPs. The analysis described in these proceedings is a search for neutrinos produced in dark matter annihilations in dwarf galaxies in the Northern Hemisphere, the M31 galaxy and the Virgo cluster, using three years of data from the completed IceCube detector.

IceCube is a cubic-kilometer neutrino detector installed in the ice at the geographic South Pole [] between depths of $1450 \mathrm{~m}$ and $2450 \mathrm{~m}$. Detector construction started in 2005 and finished in 2010, and for this analysis data taken between May 2011 and May 2014 were used, with a total of 982.6 days of livetime. Neutrino reconstruction relies on the optical detection of Cherenkov radiation emitted by secondary particles produced in neutrino interactions in the surrounding ice or the nearby bedrock. The DeepCore subarray as defined in this analysis includes 8 densely instrumented strings optimized for low energies plus 12 adjacent standard strings [3].

\section{Signal expectation}

The expected differential neutrino flux from dark matter annihilations is given by

$$
\frac{d \phi_{v}}{d E}=\frac{\left\langle\sigma_{A} v\right\rangle}{4 \pi \cdot 2 m_{\chi}^{2}} \frac{d N_{v}}{d E} J(\Delta \Omega)
$$

with $\left\langle\sigma_{A} v\right\rangle$ the velocity-averaged annihilation cross section, $m_{\chi}$ the mass of the WIMP and $d N_{v} / d E$ the WIMP annihilation spectrum. $J(\Delta \Omega)$ is the so-called (integrated) $J$-factor,

$$
J(\Delta \Omega)=\int_{\Delta \Omega} d \Omega \int_{\text {l.o.s. }} \rho^{2}(l) d l
$$

where $l$ is the coordinate along the line of sight, $\rho$ is the dark matter density and $\Delta \Omega$ is the solid angle being integrated over.

The WIMP annihilation spectrum $d N_{v} / d E$ depends heavily on the specific annihilation channels of the WIMP and their branching ratios. For this model-independent analysis, we have studied four benchmark channels (neutrinos, muons, $\mathrm{W}$ bosons and $\mathrm{b}$ quarks) which are typical for many models and in each case assumed a $100 \%$ branching ratio to this channel. For each of these channels (where possible) we have considered 19 WIMP masses ranging from $30 \mathrm{GeV}$ to $100 \mathrm{TeV}$.

For annihilation to neutrinos, $d N_{v} / d E$ is a line spectrum at $E_{v}=m_{\chi}$, since the WIMPs are assumed to annihilate at rest. For the other channels, the annihilation spectra were computed using Pythia 8.1 [四]. Since the region in which neutrinos are produced is much larger than the typical neutrino oscillation length, the spectra at the Earth can be averaged and are no longer dependent on 


\begin{tabular}{|c||c|c|c|c|c|}
\hline Targets & Type & RA [deg] & Dec $[\mathrm{deg}]$ & Distance $[\mathrm{kpc}]$ & $\log _{10}\left(J_{N F W} / \mathrm{GeV}^{2} \mathrm{~cm}^{-5}\right)$ \\
\hline \hline Segue 1 & Dwarf galaxy & 151.767 & 16.082 & 23 & $19.5 \pm 0.29$ \\
\hline Ursa Major II & Dwarf galaxy & 132.875 & 61.310 & 32 & $19.3 \pm 0.28$ \\
\hline Willman 1 & Dwarf galaxy & 162.343 & 51.051 & 38 & $19.1 \pm 0.31$ \\
\hline Coma Berenices & Dwarf galaxy & 186.746 & 23.919 & 44 & $19.0 \pm 0.25$ \\
\hline Draco & Dwarf galaxy & 260.052 & 57.915 & 76 & $18.8 \pm 0.16$ \\
\hline M31 & Major galaxy & 10.685 & 41.269 & 785 & $19.2 \pm 0.1$ \\
\hline Virgo & Galaxy cluster & 187.704 & 12.391 & 16800 & 18.5 \\
\hline
\end{tabular}

Table 1: The list of targets considered in the analysis described in these proceedings, including their locations $[\mathbb{U}]$ and their distances and $J$-factors assuming an NFW dark matter profile [ $[\mathbb{Q}, \mathbb{Q}, \mathbb{U}]$. For the Virgo cluster, no uncertainty on the $J$-factor is available.

the exact distance between the source and the Earth [可. For each annihilation channel, neutrinos of all flavours will arrive at Earth, but for this analysis only muon neutrinos are considered, so only track-like events are used.

As a consequence of equations [.] and [2.2, the expected flux is highest in regions with a high dark matter density which are relatively close by. The $J$-factor will in general depend on the dark matter halo profile, in this analysis we consider the Navarro-Frenk-White (NFW) profile [G], which is given by

$$
\rho(r)=\frac{\rho_{0}}{\frac{r}{R_{s}}\left(1+\frac{r}{R_{s}}\right)^{2}}
$$

with $\rho_{0}$ and $R_{s}$ the characteristic density and radius.

Dwarf spheroidal galaxies orbiting the Galaxy are interesting targets for indirect dark matter searches because they are close to the Earth (see table $\square$ ), highly dark-matter-dominated and have no expected astrophysical backgrounds [W]]. In this analysis, the five dwarf galaxies in the Northern Hemisphere with the highest $J$-factors are considered: Segue 1, Ursa Major II, Willman 1, Coma Berenices and Draco.

In addition to the dwarf galaxies, we also consider the M31 galaxy and the Virgo cluster. As can be seen from table W, the $J$-factors for these targets are smaller than for the best dwarf galaxies, leading to a lower sensitivity. However, from N-body simulations [ㅁ], [3] it is known that dark matter haloes for major galaxies and galaxy clusters are expected to contain a large amount of substructure, which could increase the expected flux significantly, especially at larger distances from the center of the dark matter halo. Initial estimates for the total size of this increase were of the order of 1000 for galaxy clusters [ㅍ], but later studies give more modest estimates [15], [16]. Since the subhaloes are also expected to change the spatial shape of the dark matter profile, we will give limits for the conservative case where there is no significant substructure and the dark matter profile is given by equation [2.3, but we will also search for an extended emission from the direction of M31 and the Virgo cluster. 


\section{Event selection and reconstruction}

The main backgrounds for neutrinos produced in dark matter annihilations are atmospheric muons (triggering the detector at a rate of approximately $2.5 \mathrm{kHz}$ ) and atmospheric neutrinos (approximately $20 \mathrm{mHz}$ ). Atmospheric neutrinos are an irreducible background, but atmospheric muons can be eliminated from the event sample by removing events which are either downgoing or badly reconstructed. It is also possible to remove atmospheric muons by using the outer layers of IceCube as a veto.

The first set of cuts consists of a number of online filters which select either track-like events, upgoing events or low-energy events where there is no evidence of activity in the detector which would be consistent with an atmospheric muon. These are followed by cuts which remove downgoing events, badly reconstructed events, noise events and events which do not contain enough information for a full direction reconstruction.

The sample is then split in two independent subsamples: one containing events which mostly take place in the DeepCore subdetector (the DeepCore-dominated subsample), and one containing all other events (the IceCube-dominated subsample). For each of these subsamples, a number of further cuts related to the quality of the reconstruction and the light pattern in the detector are made.

The final step of the event selection consists of a cut on the output of a Boosted Decision Tree (BDT) [ए]]. A BDT is a multivariate machine learning algorithm which is trained to separate signal and background events using training samples of both (which are discarded for the actual analysis). For the DeepCore-dominated subsample, the BDT was trained on $500 \mathrm{GeV}$ WIMPs annihilating to $\mathrm{b}$ quarks. For the IceCube-dominated subsample, one BDT was trained on $500 \mathrm{GeV}$ WIMPs annihilating to muons (the 'soft BDT') and one was trained on $10 \mathrm{TeV}$ WIMPs annihilating to muons (the 'hard BDT'). For each target, the final cut on the BDT output score is optimized for the best sensitivity to $\left\langle\sigma_{A} v\right\rangle$. Also, for each combination of a target, a WIMP mass and an annihilation channel, the sensitivities using the hard BDT and using the soft BDT are calculated. In this analysis, we choose whichever one gives the best sensitivity.

The direction reconstruction used for this analysis is described in detail in section 2.1 of [ए]8].

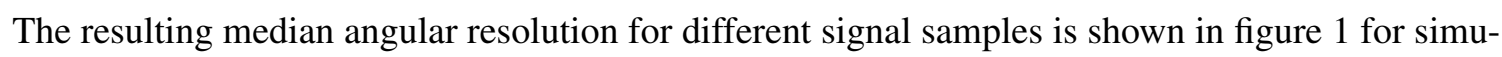
lated signal events passing the event selection up to the final level, using a typical cut on the output of the final-level BDT. A per-event estimation of the angular reconstruction error is also calculated [एव].

To reconstruct the energy of the event, different algorithms are used for the IceCube-dominated sample (which contains mainly events above $100 \mathrm{GeV}$ ) and the DeepCore-dominated sample (which contains mainly events below $100 \mathrm{GeV}$ ). For the IceCube-dominated sample, an algorithm is used which reconstructs the energy loss of the muon in the detector [20], while for the DeepCoredominated sample, a dedicated low-energy reconstruction algorithm is used [2]]. In both cases only the difference in the distributions of output values for signal and background is used, not the absolute output values.

\section{Analysis method}

After the event selection, the event sample consists mainly of atmospheric neutrinos, with 


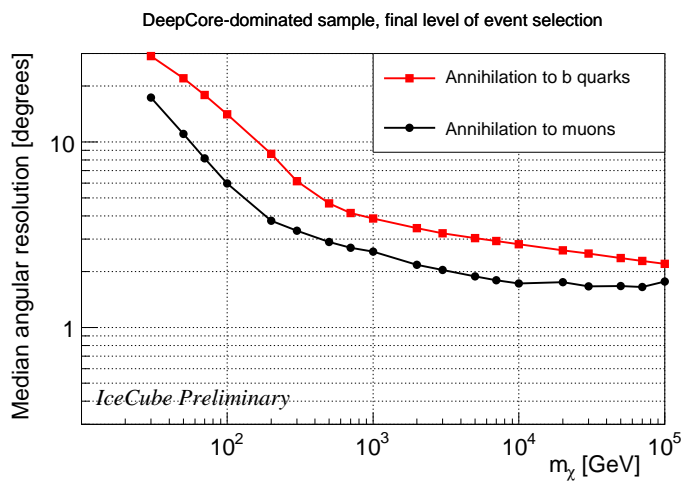

(a) DeepCore-dominated

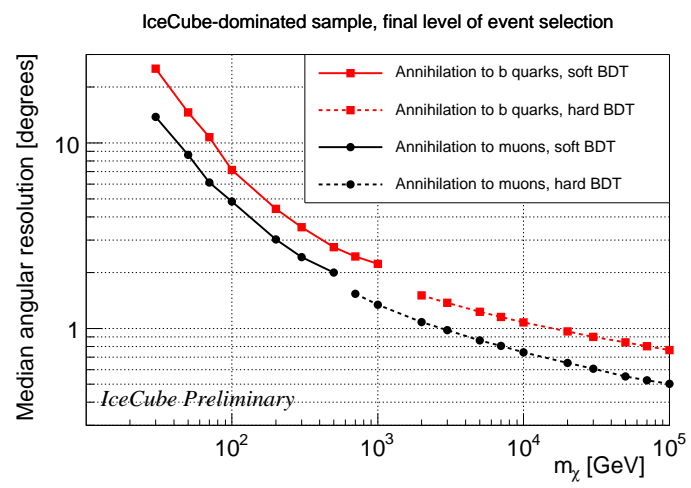

(b) IceCube-dominated

Figure 1: The median angular resolution for different WIMP masses and annihilation channels, using simulated signal events passing the event selection up to the final level, using a typical cut on the output of the final-level BDT. For (b), the sample selected by cutting on the output of the soft BDT is used for masses where the soft BDT gives the better sensitivity to $\left\langle\sigma_{A} v\right\rangle$, otherwise the sample selected by cutting on the output of the hard BDT is used.

a contamination of approximately $10 \%$ (depending on subsample and BDT cut) of atmospheric muons. To determine whether the sample contains neutrinos from dark matter annihilations taking place in the targets that are considered in this analysis, a likelihood method is used which is based on that used in [22], but takes into account the general improvement suggested in [23]. The likelihood for a single sample containing $n_{o b s}$ events, of which $\mu$ are signal events, can be written as

$$
\mathscr{L}(\mu)=\prod_{i=1}^{n_{o b s}}\left(\frac{\mu}{n_{o b s}} S_{i}+\left(1-\frac{\mu}{n_{o b s}}\right) B_{i}\right)
$$

with the signal PDF $S_{i}$ for an event with space angle (angle between the reconstructed muon direction and the target direction) $\psi_{i}$, reconstructed energy $E_{i}$ and direction reconstruction error estimate $\sigma_{i}$ given by

$$
\begin{aligned}
S_{i}\left(\psi_{i}, E_{i}, \sigma_{i}\right) & =f_{\text {sig }}\left(\psi_{i} \mid \sigma_{i}\right) \cdot g_{\text {sig }}\left(E_{i}, \sigma_{i}\right) \\
& =\frac{\psi_{i}}{\sigma_{i}^{2}} \exp \left(-\frac{\psi_{i}^{2}}{2 \sigma_{i}^{2}}\right) \cdot g_{s i g}\left(E_{i}, \sigma_{i}\right)
\end{aligned}
$$

with $f_{\text {sig }}\left(\psi_{i} \mid \sigma_{i}\right)$ the probability for signal events to have a space angle $\psi_{i}$ given that the direction reconstruction error estimate is $\sigma_{i}$, which is assumed to follow a two-dimensional Gaussian distribution with a width given by $\sigma_{i} . g_{s i g}\left(E_{i}, \sigma_{i}\right)$ is the probability for a signal event to have a reconstructed energy $E_{i}$ and error estimate $\sigma_{i}$. This is taken from simulated signal events and smoothed using Kernel Density Estimation [24, 25]].

The background PDF is given by

$$
B_{i}\left(\psi_{i}, E_{i}, \sigma_{i}\right)=f_{b g}\left(\psi_{i}\right) \cdot g_{b g}\left(E_{i}, \sigma_{i}\right)
$$


where $f_{b g}\left(\psi_{i}\right)$ and $g_{b g}\left(E_{i}, \sigma_{i}\right)$ are taken from data events where the azimuth has been randomized, and $g_{b g}$ is smoothed in the same way as $g_{s i g}$. Background events are isotropic, so $\psi_{i}$ is not correlated with $\sigma_{i}$ or $E_{i}$.

For this analysis, only events where the difference between the reconstructed zenith and the zenith of the target is less than 2.5 degrees (for the dwarf galaxies) or 5 degrees (for the M31 galaxy and the Virgo cluster) are considered, since events which are further from the target are very unlikely to be signal events. The PDFs are constructed using only events in these zenith bands, and thus are not only different for each WIMP mass and annihilation channel (in case of the signal PDFs) but also for each target.

For multiple independent subsamples, equation 4.1 is adapted to

$$
\mathscr{L}(\mu)=\prod_{j=1}^{n_{\text {samp }} n_{\text {obs }, j}} \prod_{i=1}\left(\frac{\mu_{j}}{n_{o b s, j}} S_{j, i}+\left(1-\frac{\mu_{j}}{n_{o b s, j}}\right) B_{j, i}\right)
$$

where $j$ runs over the different subsamples, the PDFs are now (in general) different for each sample, and the total number of signal events in all subsamples combined is $\mu=\sum_{j} \mu_{j}$.

For this analysis, the DeepCore-dominated and IceCube-dominated subsamples are independent, as are the three years of data-taking. We also stack the five dwarf galaxies by considering them as subsamples, which are indeed independent since the zenith bands do not overlap.

We obtain a best estimate for $\mu$ for each WIMP mass and annihilation channel by maximizing the likelihood given in equation 4.4. Using the method of Feldman and Cousins [[26], confidence intervals on the number of signal events present in the total sample and thus on the velocity-averaged annihilation cross section can be constructed.

As mentioned in the introduction, we also search for a more extended emission from M31 and the Virgo cluster, which could be caused by dark matter substructure. As the specific profile and extension are unknown, we consider two-dimensional Gaussian source profiles with widths 0.5 , 1,2 and 5 degrees. In this case, the two-dimensional Gaussian distribution used as $f_{\text {sig }}\left(\psi_{i} \mid \sigma_{i}\right)$ in equation 4.2 is convolved with the source profile, and the result is used as $f_{s i g}\left(\psi_{i} \mid \sigma_{i}\right)$ instead.

\section{Results and conclusion}

For the first time, data from the completed IceCube detector has been used to search for neutrinos produced in dark matter annihilations in nearby extra-Galactic objects. No significant excess of neutrinos was found in the direction of any of the targets that were considered, and upper limits at the $90 \%$ confidence level were calculated. In figure 2a the sensitivities and limits are compared for the stacking of five dwarf galaxies, the M31 galaxy and the Virgo cluster, for annihilation to muons. In figure $[\mathrm{b}$, the limits for different annihilation channels are compared.

Since the background for this analysis was determined directly from data, only systematic uncertainties on the signal acceptance play a significant role. These are dominated by the uncertainty on the sensitivity of the PMTs used for detecting the Cherenkov light and the optical properties (scattering and absorption lengths) of the ice. To get the total systematic uncertainty, these are varied by $10 \%$ and the resulting uncertainties are added in quadrature. The total systematic uncer- 


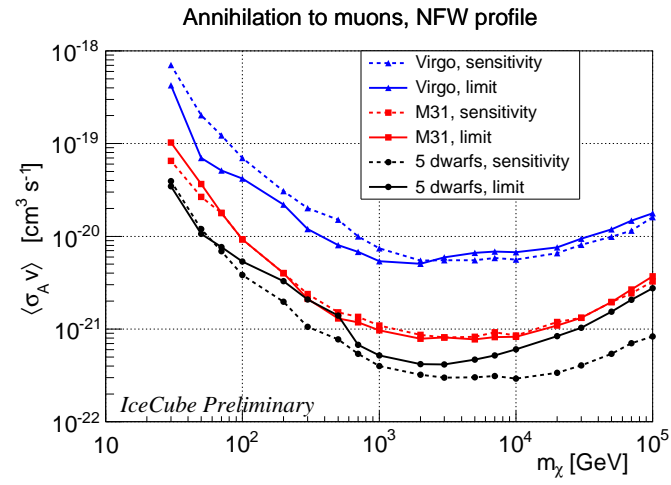

(a) Comparison of upper limits and sensitivities for the stacking of five dwarf galaxies, the M31 galaxy and the Virgo cluster, for annihilation to muons.

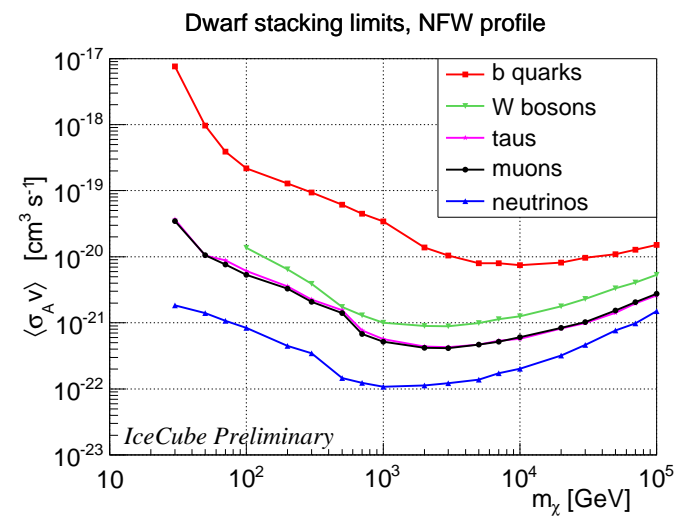

(b) Comparison of upper limits for the different annihilation channels considered in this analysis, for the stacking of five dwarf galaxies.

Figure 2: The $90 \%$ confidence limits on the velocity-averaged annihilation cross section.

tainty which is included in the limits ranges from approximately $15 \%$ to $35 \%$, depending on the source, WIMP mass and annihilation channel.

For the stacking of five dwarf galaxies, there is an overfluctuation for high WIMP masses which shows up for all channels and a broad range of masses, as the PDFs look relatively similar. To estimate the global significance of this excess, a test statistic is used which is defined as

$$
T S=2 \log \frac{\mathscr{L}(\hat{\mu})}{\mathscr{L}(0)}
$$

with $\hat{\mu}$ the best fit value for $\mu$. A large number of pseudo experiments using data events with randomized azimuths were performed, and for $4.3 \%$ of these background-only pseudo experiments, there was at least one WIMP mass and channel combination with a higher $T S$. This makes it clear that the excess is not statistically significant.

Searching for an extended neutrino emission from M31 and the Virgo cluster also led to no significant excess, so all results of this analysis are compatible with the background-only hypothesis.

\section{References}

[1] G. Bertone, D. Hooper, and J. Silk, Physics Reports 405 (2005) 279-390.

[2] IceCube Collaboration, A. Achterberg et al., Astroparticle Physics 26 (2006), no. 3 155-173.

[3] IceCube Collaboration, R. Abbasi et al., Astroparticle Physics 35 (2012), no. 10 615-624.

[4] T. Sjöstrand, S. Mrenna, and P. Skands, Computer Physics Communications 178 (2008), no. 11 852-867.

[5] S. Bilenky and S. Petcov, Reviews of Modern Physics 59 (1987), no. 3 671-754.

[6] J. Navarro, C. Frenk, and S. White, The Astrophysical Journal 490 (1997), no. 2493.

[7] SIMBAD catalog, http://simbad.u-strasbg.fr/simbad/. 
[8] Fermi-LAT Collaboration, M. Ackermann et al., Physical Review D 89 (2014), no. 4042001.

[9] A. Tamm et al., Astronomy \& Astrophysics 546 (2012) A4.

[10] J. Han et al., Monthly Notices of the Royal Astronomical Society 427 (2012), no. 2 1651-1665.

[11] M. Mateo, Annual Review of Astronomy and Astrophysics 36 (1998) 435-506.

[12] V. Springel et al., Monthly Notices of the Royal Astronomical Society 391 (2008), no. 4 1685-1711.

[13] J. Diemand et al., Nature 454 (2008), no. 7205 735-738.

[14] L. Gao et al., Monthly Notices of the Royal Astronomical Society 419 (2012), no. 2 1721-1726.

[15] D. Anderhalden and J. Diemand, Journal of Cosmology and Astroparticle Physics 2013 (2013), no. 04 009.

[16] M. Sánchez-Conde and F. Prada, Monthly Notices of the Royal Astronomical Society 442 (2014), no. 3 2271-2277.

[17] Y. Freund, R. Schapire, and N. Abe, Journal - Japanese Society for Artificial Intelligence 14 (1999).

[18] IceCube Collaboration, M. Aartsen et al., The Astrophysical Journal 796 (2014), no. 2109.

[19] T. Neunhöffer, Astroparticle Physics 25 (2006), no. 3 220-225.

[20] IceCube Collaboration, M. Aartsen et al., Journal of Instrumentation 9 (2014), no. 0303009.

[21] IceCube Collaboration, M. Aartsen et al., Physical Review D 91 (2015), no. 7072004.

[22] J. Braun et al., Astroparticle Physics 29 (2008), no. 4 299-305.

[23] G. Punzi, Comments on likelihood fits with variable resolution, 2004. arXiv:physics/0401045.

[24] M. Rosenblatt, The Annals of Mathematical Statistics 27 (1956), no. 3 832-837.

[25] E. Parzen, The Annals of Mathematical Statistics 33 (1962), no. 3 1065-1076.

[26] G. Feldman and R. Cousins, Physical Review D 57 (1998), no. 73873. 